\title{
The Monarch Room: An Interactive System for Visualization of Global Migration Data
}

\author{
Ana Caraban \\ Madeira-ITI \\ Funchal, Portugal \\ ana.caraban@m-iti.org
}

\author{
Teresa Paulino \\ Madeira-ITI \\ Funchal, Portugal \\ teresa.paulino@m-iti.org
}

\author{
Ricardo Pereira \\ Madeira-ITI \\ Funchal, Portugal
}

\author{
Robert Spence \\ Imperial College London \\ London, England
}

\author{
Pedro Campos \\ Madeira-ITI \\ Funchal, Portugal
}

\begin{abstract}
Representation, presentation and interaction are subjects that require to be consciously contemplated while designing information visualization systems. This paper describes the ideation and design process of The Monarch Room, an information visualization system of global migration data. Our solution was designed to allow visitors of a museum to select, browse and interact with a visual interactive representation of migration flows in a unified view. Visitors can convey their learning purposes by seamlessly combining criteria such as time scales, location, and reasons behind migration, from either manual our automatic interactions. We believe our work contributes to the design of interfaces that enable a more in-depth understanding of global human migration patterns. In this paper, we describe our first design and research efforts.
\end{abstract}

Data visualization, Interactive System, Spherical Screen, Museum Room Prototype, Design Process, Interaction Design, World Migration.

\section{INTRODUCTION}

To keep a museum's reputation, it is important to capture visitors' attention while offering them valuable insights and experiences. This necessity has led to a growing interest in the use of interactive technology in a museum setting. Installations that make use of animations and visual effects, such as projections, have the potential of providing engaging and enjoyable experiences. They are well suited for communicating information, promoting visitors engagement, supporting sensemaking, and engaging conversations during their visit [1]. Yet while many interactive exhibitions exist, they still face some constrains, such as low engagement, poor support for simultaneous interaction and the provision of interfaces that are easy to use and to understand. As designers, creating interactive exhibits that are able to present detailed information presents a challenge: How can we create a visualization that allows visitors to understand so much data? In this work, we describe our early research efforts on the design of the Monarch Room, an interactive exhibit designed to help visitor learns about migration data. We envisioned an interactive room that takes advantage of a spherical screen as a platform that presents global human migration visualizations while offering visitors contextual information related with a specific event or time. Our aim is not only to engage visitors with the exhibition but also to support users in their learning goals. We report on the detailed design process and we conclude with a list of additional features that we intent to incorporate in future iterations.

\section{RELATED WORK}

\section{Spatial interactions and Interactive Interfaces}

Spatial Interactions are among the essential forces that drive many socioeconomic processes. Described as location-to-location movements of human beings (e.g. intra urban or intercontinental commuters) or traffic in goods, these dynamic flow processes are often an important factor in a wide range of fields such as business or government, which requires taking the right decision based on the available data [12]. Due to it repercussion, the use of spatial data if often employed in culturalbased settings, such as museums, as it enables the understanding of many historical processes that drive social, behavioral or economic phenomena.

One might notice that flow maps are the main approach used to visualize spatial interaction data $[12,14,15]$. One limitation is that geographically embedded networks are usually very large and often involve thousands of locations and connections between nodes (see Figure 1) [12]. In addition, another constrain is the amount of variables associated with data flow (e.g. number of nomads, personal information such as age, occupation, among others) [12]. It is a challenge to visualize such a large amount of data and obtain insights simultaneously. 

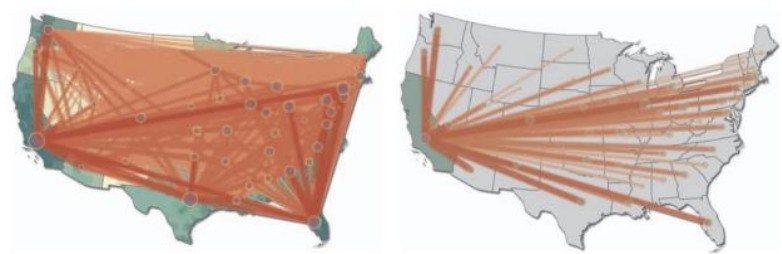

Figure 1 - Country migration data in the U.S [12].

In response, the design of interactive visualizations has been explored to overcome these limitations. For instance, D3.js and Tableau two interactive data visualizations tools [13], allows the manipulation of data properties, which help users to identify patterns on the amount of information available through visual encoding, interaction and animations. Yet, despite its potential, this approach does not allow simultaneous interaction (i.e. multiple users) and has not been explored in physical scenarios to investigate how it can enhance visitor's engagement and immersion. We envisioned a comparable interactive visualization on an interactive surface.

Prior work has demonstrated that information presented on interactive surfaces offer plausible opportunities for interactions in public spaces. Interactive exhibits not only attracts and holds visitor's attention but also prompts visitors interactions which are set as more immersive $[5,17,18]$. They can promote physical engagement, prompt reflection and incite social collaboration in learning environments in a way that exhibit's visitors can gather and debate around the surface. We believe that employing compelling and dynamic content through an interactive surface can capture visitors' attention while offering them valuable insights and experiences.

In this work, we address previously mentioned challenges by designing an interactive visualization display that consists of three coupled components: (1) an interactive multi touch spherical screen that displays geographical regions and corresponding flow connections in a walk-up-and-use setting, (2) an input method embedded in the spherical screen that infers users requests and adjusts flow patterns accordingly, and a (3) projection system that complements migration data by providing audiovisual information on the walls of the exhibition.

\section{DESIGN PROCESS - THE MONARCH ROOM}

In this section, we present and describe the conceptual system and features idealized after performing brainstorming, converging and sketching sessions.

3.1 Spherical screen -The Monarch Room was designed as an interactive system for visualization of global migration data. To facilitate the understanding of migration through different countries, we emulated the globe through a spherically shaped screen located in the middle of the museum exhibit, which can be rotated left and right around a vertical axis (see Figure 5). To allow dynamic interactions, the sphere is devised as multi touch display, which allows users to explore flow connections in a walk-up-and-use setting.

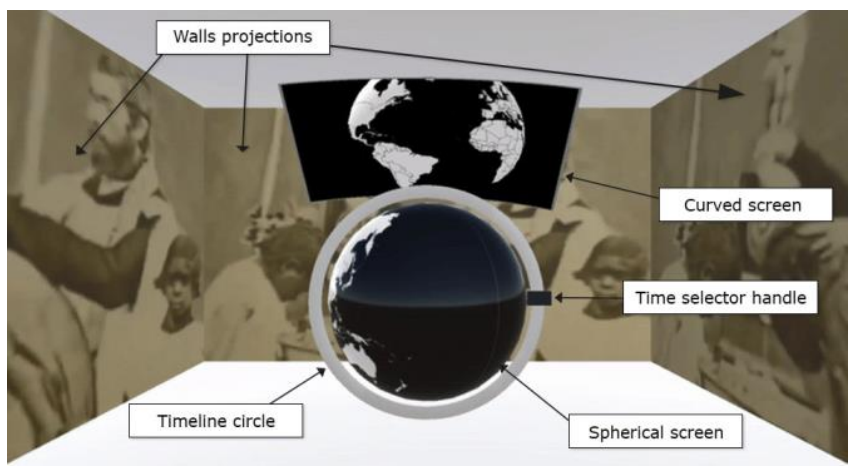

Figure 2 - Simple 3D representation of The Monarch Room

3.2 Time selection - To enable time selection, restricting the information displayed, we envisioned a "timeline ring" located around the spherical screen that not only holds the screen but also comprises two handles that enable time selection. The handles can be moved across the ring and their position identifies the timeframe selected. For instance, when both handles are placed together (i.e. one underneath the other) the system assumes that the time selection is for a specific year. In turn, when handles are placed separately, the system assumes a time period (see Figure 3), which is inferred through the location of the handles across the ring.

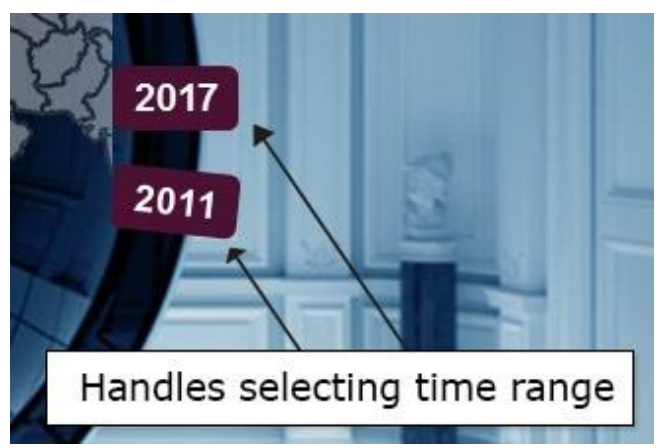

Figure 3: Time selection. Two handles allow the selection of one specific time or time range.

3.3 Contextual filters - To provide a more insightful and personalized information to the user, the system allows users to filter the information by distinctive categories (Figure 4Figure 4). Placing magnetic tags, available close to the spherical screen, enables data filtering. Each magnetic tag has its own colour and shape and corresponds to a filter category. It is possible to combine tags 
(attached on top of each other), which may provide a more insightful visualization (see Figure 4).

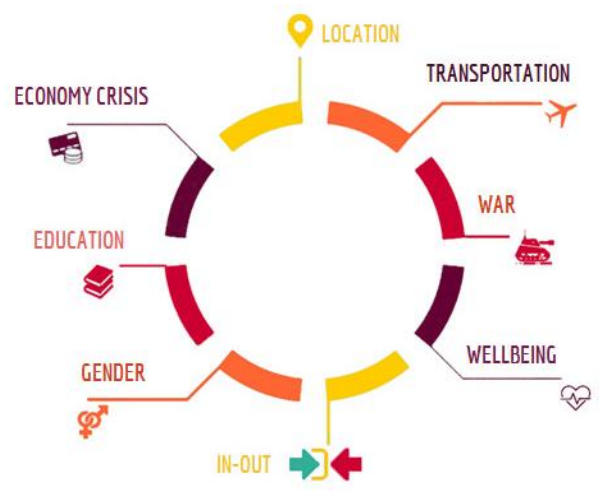

Figure 4: Contextual filters to allow a visitor to work with a specific subset of data.

3.4 Representation of people movements - To represent migration movements, people's journeys are represented by lines, which can be distinguished by colour and thickness. Colours represent in and out movements. Thickness in turn, offers quantitative representation of the amount of people, where dashed lines represent less, and thicker lines represent more people moving.

3.5 Back view display - Since the sphere has a round shape and all the information may not be visible from one point of view, our concept includes one or more curved screens hanging on the top of the wall to display the opposite side of the sphere. This feature enables users to be aware of information being displayed on the other side of the globe.

3.6 Walls' projections - Moreover, the interactive system can project audio-visual information on the walls if one or more magnetic tags (i.e. filters) are applied. The content displayed relates to the time range, country and selected filter, enhancing visitor's engagement and immersion. For instance, providing videos of news reports across time.

3.7 Virtual Reality Application - To create a memorable experience, we envision this system to be complemented with a virtual reality (VR) application provided as a gift for visitors, which could be also available on the museum's website. This feature will enable users to experience The Monarch Room from any part of the world, which could also be used for educational purposes.

\section{SCENARIO OF INTERACTION}

To perceive the helpfulness of our approach understanding migration data, we tested our approach in a semi-formal environment. We invited universities researchers and students to join a project presentation and collected qualitative data from attendee's thoughts using a think-aloud method. We designed and simulated our system using Unity 3D engine and an animated PowerPoint presentation (see Figure 2 and 3). To provide a better understanding of a possible situation of interaction, we present Paul, an archetypal user that is visiting the museum where the interactive system is installed and presented the following narrative "Paul enters The Monarch Room, the spherical screen at the centre is displaying global migration information, automatically progressing in time. Paul comes closer to the sphere and touches the time handle. The system switches to manual mode and the visualization is adapted to the year that the handle was selecting when Paul touched it: 2011. Paul drags the handle and notices there are two handles, one was underneath the other. The data being visualized refers now to a range of time, from 2011 to 2017. Paul notices the box of magnetic tags close to the sphere and chooses the location tag. Paul decides to place the location tag in Syria, the spherical screen reacts to the presence of a magnetic tag and the information instantly changes (see Figure 5Error! Reference source not found.). The lines refer now only to Syria country, the lines thickness help Paul to quickly gain and insight about the people 'movements. Just for curiosity Paul places the "war" tag over the previous tag, Paul knew about Syria situation so was not surprised by not seeing much change on the data being presented: most of the movements are war related. When Paul placed the war tag, the walls of The Monarch Room started to display videos and images about the war in Syria. Paul got even more immersed in the context of migration being presented at the room. By looking at the curved screen hanging on the top of the wall, Paul could notice that the Syrian movements were all around the globe, what led him to rotate the sphere to see the full lines extension. Paul spent a while in the room, exchanging tags and choosing various locations, what ended up by being a playful experience. At the end of that day, the museum offered him a mobile VR app, which will enable him to experience The Monarch Room from home and share it with friends and family."

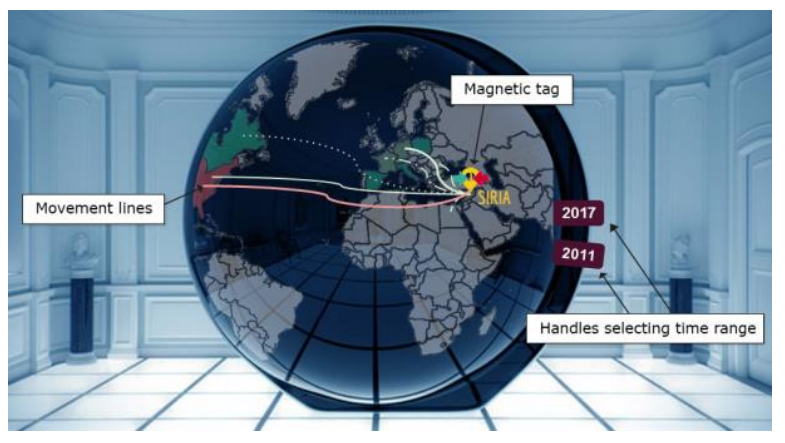

Figure 5 - The spherical screen displaying the lines that represent people movements in Syria within the time range 2011-2017 
After the session we transcribed attendee's thoughts. Data gathered during the think-aloud protocol allowed us to identify future design considerations of The Monarch Room including:

1. The enhancement of the lines to provide more distinction of the presented information and make the information visible up to a certain distance.

2. The use of more filters, conveying the type of migrant (e.g. refugees) and the addition of information depicting the causes and effects that triggered a relevant migration event. We observed that participants were interested on selecting and combining different filters in order to obtain tailored information during specific timeframes, which usually required demanding amount of time and effort on other digital platforms (e.g. websites).

3. The possibility to display audio-visual content displayed on the spherical screen instead of on the walls. We observed that participants were more interesting on distinguishing and learning facts through the interactive visualization.

\section{CONCLUSION}

In this paper we present the design process of an information visualization system aimed to help visitors of a museum to gain a better understanding about global migration while promoting their engagement. We defined what and how information is represented and distinguished on the system, how content is presented to the final user, and what type of interactions may occur. By implementing design for systematic approaches, our solution may be improved to achieve a broader purpose than just migration data, reaching other museums with diverse cultural thematic. Our future work aims to explore different hardware components to reduce the overall cost of the system and explore the use of narratives tailored to the target audience (e.g. if a school is visiting the museum, the system can display stories adapted to a specific age range, increasing their engagement with the story.

\section{ACKNOWLEDGEMENTS}

This research was supported by ARDITI under the scope of the Project M1420-09-5369-FSE-000001 PhD Studentship.

\section{REFERENCES}

[1] D. A. Basballe and K. Halskov, 'Projections on Museum Exhibits: Engaging Visitors in the Museum Setting', in Proceedings of the 22nd Conference of the Computer-Human Interaction Special Interest Group of Australia on Computer-Human Interaction - OZCHI '10, 2010 , p. 80.
[2] G. Hakvoort and Gido, 'The immersive museum', in Proceedings of the 2013 ACM international conference on Interactive tabletops and surfaces - ITS '13, 2013, pp. 463-468.

[3] J. Rodrigues, C. M. Q. Ramos, P. J. S. Cardoso, C. Henriques, and IGI Global, Handbook of research on technological developments for cultural heritage and eTourism applications. .

[4] J. E. Slentz, 'Yes, You May Touch the Art: New Media Interfaces and Rhetorical Experience in the Digitally Interactive Museum', 2017.

[5] C. Sandifer, 'Technological novelty and openendedness: Two characteristics of interactive exhibits that contribute to the holding of visitor attention in a science museum', J. Res. Sci. Teach., vol. 40, no. 2, pp. 121-137, Feb. 2003.

[6] H. Benko, A. D. Wilson, and R. Balakrishnan, 'Sphere: multi-touch interactions on a spherical display', in Proceedings of the 21st annual ACM symposium on User interface software and technology - UIST '08, 2008, p. 77.

[7] H. Benko and Hrvoje, 'Beyond Flat Surface Computing: Challenges of Depth-aware and Curved Interfaces', in Proceedings of the seventeen ACM international conference on Multimedia - MM '09, 2009, p. 935.

[8] J. R. Williamson, D. Sundén, and J. Bradley, 'GlobalFestival: Evaluating Real World Interaction on a Spherical Display', in Proceedings of the 2015 ACM International Joint Conference on Pervasive and Ubiquitous Computing - UbiComp '15, 2015, pp. 12511261.

[9] J. R. Williamson and D. Sundén, 'Enter the Circle: Blending Spherical Displays and Playful Embedded Interaction in Public Spaces', in Proceedings of the 4th International Symposium on Pervasive Displays - PerDis '15, 2015, pp. 195-200.

[10] M. M. Biskjaer, P. Dalsgaard, and K. Halskov, 'Creativity methods in interaction design', Proceedings of the 1st DESIRE Network Conference on Creativity and Innovation in Design. Desire Network, pp. 12-21, 2010.

[11] R. Spence, 'Information Visualization: an Introduction', in Information Visualization, Cham: Springer International Publishing, 2014.

[12] Guo, D. (2009). Flow mapping and multivariate visualization of large spatial interaction data. IEEE Transactions on Visualization and Computer Graphics, 15(6). 
[13] Nair, L. R., Shetty, S. D., \& Shetty, S. D. (2016). Interactive visual analytics on Big Data: Tableau vs D3. js. Journal of e-Learning and Knowledge Society, 12(4).

[14] W.R. Tobler, Spatial Interaction Patterns, Journal of Environmental Systems, vol. 6, no. 4, pp. 271-301, 1976.

[16] W.R. Tobler, Experiments in Migration Mapping by Computer, American Cartographer, vol. 14, pp. 155-163, 1987.

[17] Cesário, V., Radeta, M., Matos, S., \& Nisi, V. (2017, October). The Ocean Game: Assessing Children's Engagement and Learning in a Museum Setting Using a Treasure-Hunt Game. CHI Play pp. 99-109

[18] Cesário, V., Radeta, M., Coelho, A., \& Nisi, V. (2017, September). Shifting from the Children to the Teens' Usability: Adapting a Gamified Experience of a Museum Tour. Interact (pp. 464-468). 